\title{
Beyond gut microbiota: understanding obesity and type 2 diabetes
}

\author{
Eva Lau, ${ }^{1}$ Davide Carvalho, ${ }^{1}$ Cidália Pina-Vaz, ${ }^{2}$ José-Adelino Barbosa, ${ }^{3}$ Paula Freitas ${ }^{1}$ \\ ${ }^{1}$ Department of Endocrinology, Diabetes and Metabolism, Centro Hospitalar São João; Faculty of Medicine University \\ of Porto; Instituto de Investigação e Inovação em Saúde, Universidade do Porto; ${ }^{2}$ Department of Microbiology, Centro \\ Hospitalar São João; Faculty of Medicine University of Porto; CINTESIS, Center for Health Technology and Services Research \\ Porto; ${ }^{3}$ Department of Surgery, Centro Hospitalar São João; Faculty of Medicine University of Porto; Porto, Portugal
}

\begin{abstract}
Obesity and type 2 diabetes are metabolic diseases that have reached epidemic proportions worldwide. Although their etiology is complex, both result from interplay between behaviour, environment and genetic factors. Within ambient determinants, human overall gut bacteria have been identified as a crucial mediator of obesity and its consequences. Gut microbiota plays a crucial role in gastro-intestinal mucosa permeability and regulates the fermentation and absorption of dietary polyssacharides, which may explain its importance in the regulation of fat accumulation and the resultant development of obesity-related diseases. The main objective of this review is to address the pathogenic association between gut microbiota and obesity and to explore related innovative therapeutic targets. New insights into the role of the small bowel and gut microbiota in diabetes and obesity may make possible the development of integrated strategies to prevent and treat these metabolic disorders.
\end{abstract}

Key words: Diabetes, Gut microbiota, Obesity

\section{INTRODUCTION}

Overweight and obesity are metabolic diseases that have spread worldwide, today reaching epidemic proportions and thus representing the fifth leading risk factors for global mortality. At least 2.8 million adults die each year as a result of being overweight

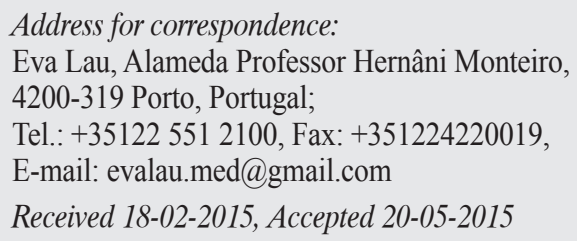

or obese. In addition, $44 \%$ of diabetes cases, $23 \%$ of ischemic heart disease cases and between $7 \%$ and $41 \%$ of certain cancer burdens are attributable to overweight and obesity. ${ }^{1}$ Type 2 diabetes (T2D) is today following the trend of obesity and increasing globally. It is estimated that 347 million people worldwide have diabetes. ${ }^{2}$ Although the etiology is complex, both obesity and diabetes result from interplay between behaviour, environment and genetic factors, while mutations seem to be responsible for less than 10\% of phenotype variability. Thus, environmental factors stand out as the principal contributors to the obesity and diabetes epidemic. Within ambient determinants, 
human overall gut bacteria have been is thought to be a crucial mediator of obesity and diabetes pathogenesis. ${ }^{3}$ This new proposed model, the 'metagenome hypothesis', is based on the fact that humans host $10^{14}$ bacteria in the gut, representing an 150 -fold of our eukaryotic nuclear genome. ${ }^{4}$ This "microbial organ" performs a variety of physiological functions, from protective to metabolic regulation, including an active part in glucose and lipid metabolism. ${ }^{5}$ New insights into the role of the small intestine and gut microbiota in diabetes and obesity are essential for the development of innovative therapeutic targets for the prevention, treatment and delay of obesity, T2D and metabolic associated disorders.

\section{GUT MICROBIOTA}

Gut microbiota is mainly composed of seven bacterial divisions, namely, Firmicutes, Bacteroides, Proteobacteria, Fusobacteria, Verrucomicrobia, Cyanobacteria and Actinobacteria. Firmicutes and Bacteroides are the most abundant species. ${ }^{6}$ Gut microbiota performs essential functions, critical for maintenance of health, namely metabolic roles, including vitamin production, amino acid synthesis and bile acid biotransformation, protective actions preventing pathogenic colonization and structural and histological functions regulating intestinal structure and function. ${ }^{7}$ Dysbiosis is a state characterized by alteration in microbiota composition, a change in bacterial metabolic activity and/or a shift in local distribution of communities. At present, intestinal dysbiosis is crucial in understanding the pathophysiology of several metabolic diseases including that of obesity and T2D (Figure 1).

\section{Obesity and gut microbiota}

Ley et al were the first to analyze bacterial 16S rRNA gene sequences from the distal intestinal (cecal) microbiota of genetically obese ob/ob mice and lean ob/+, all fed the same polysaccharide-rich diet. They found that ob/ob animals have a 50\% reduction in the quantity of Bacteroidetes and a proportional increase in Firmicutes. ${ }^{8}$ Furthermore, this trait is transmissible: colonization of germ-free mice with an 'obese microbiota' results in a significantly greater increase in total body fat and insulin resistance than colonization with a 'lean microbiota'. ${ }^{9}$ These results suggest that obesity is linked to a different gut micro-

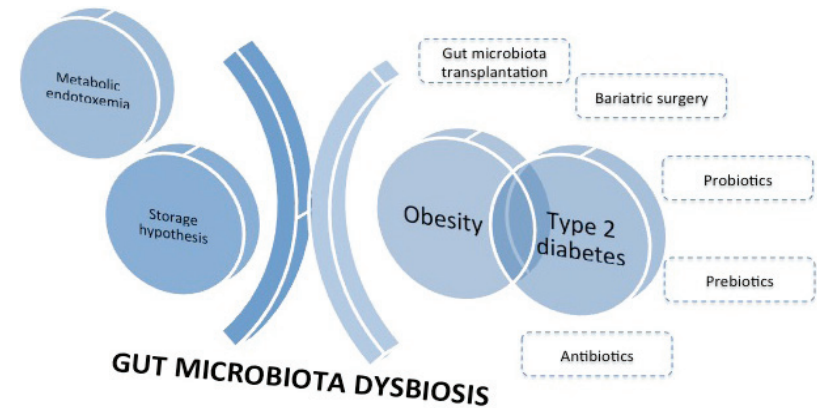

Figure 1. The pathogenic association between gut microbiota and obesity and type 2 diabetes and related innovative therapeutic targets that may treat these metabolic disturbances.

biota profile, characterized as intestinal dysbiosis. In addition, they have enabled identification of the gut microbiota as an additional contributing factor to the pathophysiology of obesity and insulin resistance. Subsequently, several studies have characterized gut microbiota in obese subjects. In obese humans, the relative proportion of Bacteroidetes was also found to be decreased by comparison with lean people. ${ }^{10}$ The same authors demonstrated a shift toward higher relative abundance of Bacteroidetes and decreased number of Firmicutes in obese patients losing weight through low-calorie diets.

One type of bariatric surgery, namely, Roux-enY gastric bypass (RYGB), has become a promising treatment for obesity, with double benefits: weight loss and metabolic improvement. Furet et al have profiled gut microbiota from fecal samples in 13 lean control subjects and in 30 obese individuals before and after RYGB. The Bacteroides/Prevotella group was lower in obese subjects before and increased 3 months (M3) after RYGB; Escherichia coli species also increased at M3 and were inversely correlated with fat mass and leptin levels, independently of food intake changes. Also, lactic acid bacteria including the Lactobacillus/Leuconostoc/Pediococcus group and Bifidobacterium genus decreased at M3. ${ }^{11}$

In a group of obese or overweight subjects who underwent a 6-week program of energy restriction, followed by another 6-week weight stabilization period, subjects who lost less weight and thus more rapidly regained it had higher Lactobacillus/Leuconostoc/ Pediococcus numbers in fecal samples at baseline. ${ }^{12}$ The gut microbiota profile may, in the near future, 
enable the prediction of which obese subjects will lose weight in response to an energy restriction diet, which is likely to be a helpful strategy in obesity treatment.

Furthermore, it was shown that low bacterial richness was linked to more marked overall adiposity and that obese adults who had lower bacterial richness had gained more weight over a 9 -year follow-up period. ${ }^{13}$

\section{Type 2 diabetes and gut microbiota}

$\mathrm{T} 2 \mathrm{D}$ is a metabolic disease characterized by a state of insulin resistance and low-grade inflammation. Microbial ecology can be an important regulator of energy homeostasis and glucose metabolism. Although animal experiments show clear differences between diabetic and non-diabetic gut microbiota, the huge variability in humans most likely masks these largescale differences.

Diabetic leptin-resistant mice $(\mathrm{db} / \mathrm{db})$ presented a higher abundance of Firmicutes, Proteobacteria and Fibrobacteres phyla compared to lean mice. ${ }^{14}$ In humans, there is lack of uniformity in the gut microbiota profile in type 2 diabetic patients. Larsen et al have studied the fecal bacterial composition of 36 adult males, among whom 18 had T2D. The proportions of Firmicutes phylum and of the Clostridia class were significantly reduced in the diabetic group compared to the control group. Furthermore, they have found that the ratios of Bacteroidetes to Firmicutes and the ratios of the Bacteroides-Prevotella group to the $C$. coccoides-E. rectale group correlated positively with glucose plasma levels. Similarly, Betaproteobacteria was highly enriched in diabetic compared to nondiabetic persons and also positively correlated with glucose plasma. ${ }^{15}$

A protocol for a metagenome-wide study in 345 type 2 diabetic Chinese individuals was recently developed. ${ }^{16}$ Type 2 diabetic patients had increased opportunistic pathogens, such as Bacteroides caccae, Clostridium hathewayi, Clostridium ramosum, Clostridium symbiosum, Eggerthella lenta and Escherichia coli, and a decreased number of some types of butyrate-producers. An interesting finding was the moderate degree of this T2D-related dysbiosis: only $3.8 \pm 0.2 \%$ (mean 6 s.e.m.; $n=5344$ ) of the gut microbial genes (at the relative abundance level) was associated with T2D in an individual. ${ }^{16}$ This observa- tion has raised the concept of a 'functional dysbiosis' rather than a specific microbial species association with T2D pathophysiology.

Karrisson et al have analyzed the gut metagenome from normal women with impaired glucose tolerance and diabetic women. ${ }^{17}$ In the total cohort, Lactobacillus species correlated positively with fasting glucose and glycated hemoglobin (HbA1c) and Clostridium species correlated negatively with fasting glucose, $\mathrm{HbA} 1 \mathrm{c}$, insulin, C-peptide and plasma triglycerides, and positively with adiponectin and HDL. Based on sets of metagenomic clusters (MGCs), they found 26 differentially abundant clusters when comparing diabetics to subjects with normal glucose tolerance. They have also created a random forest model to examine whether microbiota composition can identify diabetes status. MGCs more accurately identified T2D (highest AUC 0.83) than microbial species (highest AUC 0.71). ${ }^{17}$

One type of bariatric surgery, namely RYGB, has also made possible a better understanding of the impact of gut microbiota modification and adaptation after the procedure. ${ }^{11}$ Fecalibacterium prausnitzii species were lower in subjects with diabetes and associated negatively with inflammatory markers before and after the surgery and during the follow-up period, independently of changes in food intake. ${ }^{11}$ In humans, blood 16S rDNA, a broadly specific bacterial marker, was found to be an important independent marker of the risk of diabetes and also predicted abdominal adiposity in a large sample of non-obese participants from a general population. ${ }^{18}$ This study reaffirms the concept that tissue bacteria are involved in the onset of diabetes in humans.

\section{Mechanisms linking gut microbiota dysbiosis to obesity and type 2 diabetes}

\section{Metabolic endotoxemia}

One of the mechanisms proposed to explain the crosstalk between gut microbiota, regulation of fat storage and development of obesity-related diseases is metabolic endotoxemia. The concept described as "metabolic endotoxemia" was first described in mice. ${ }^{19,20}$

Bacterial lipopolysaccharides (LPS) are a component of the cell wall of gram-negative bacteria capable 
of triggering an inflammatory state, which is present in metabolic disorders - the metabolic inflammation hypothesis. It was shown that high-fat feeding augments plasma LPS-containing microbiota at a concentration sufficient to increase body weight, fasting glycemia and inflammation. ${ }^{19}$ Moreover, oligofructose supplementation, which increases Bifidobacteria content, reduced the inflammatory tone, namely endotoxemia, plasma and adipose tissue proinflammatory cytokines, this strongly pointing to LPS as in important mediator of inflammatory response. ${ }^{20}$

\section{The LPS/CD14/TLR4 system}

LPS in combination with CD14 serves as a ligand for toll-like receptor (TLR4). It was observed that CD14 knock-out mice, lacking functional LPS receptors, were hypersensitive to insulin. Obesity and diabetes were also delayed in response to highfat feeding. ${ }^{19}$ Furthermore, increased endotoxemia was associated with increased CD14 expression and increased IL-6 levels after a mixed meal containing lipids in healthy humans. ${ }^{21}$ Accordingly, TLR4 inactivation reduced food intake and inflammatory response, despite no significant modification of body weight. ${ }^{22}$ It also blunted insulin resistance induced by LPS in differentiated adipocytes. Thus, the LPS/ CD14/TLR4 system seems to set the threshold at which a high-fat diet induced insulin resistance and the onset of diabetes and obesity.

\section{Mucosa permeability}

Intestinal mucosa has an important role in the absorption of vital nutrients and in the regulation of barrier functions, thus preventing bacterial translocation. Intestinal mucosa integrity is ensured through intercellular tight junctions, mucus secretion, release of antimicrobial peptides from Paneth cells and immunoglobulin secretions from resident immune cells. Zonulin - a protein of the haptoglobin family released from liver and intestinal epithelial cells - is the main physiological regulator of intercellular tight junctions. Increased zonulin concentrations are related to changes in tight junction competency and increased GI permeability. ${ }^{23}$ This "leak" in the paracellular absorption route enables invasion of antigens from the intestinal milieu, triggering an immune response and subsequent inflammation and oxidative stress. ${ }^{24,25}$
A randomized, 14-week, double-blind, placebocontrolled trial with 23 endurance-trained men who took a supplement including six probiotic strains showed a significant reduction in fecal excretion of epithelial tight junction protein zonulin in response to treatment, suggesting increased integrity of the intestinal mucosa. ${ }^{26}$ Similarly, a 9-week intervention trial in 93 obese volunteers supplemented with a prebiotic showed concomitant changes in the intestinal microbiota and a reduction in intestinal permeability, assessed with a dual-sugar-absorption test. ${ }^{27}$ Increased plasma concentrations of zonulin have also been reported in a cohort of 25 patients with sepsis compared with a healthy control group, stressing the potential role of tight junction proteins in sepsis, resulting in disruption of the structural integrity of the intestinal mucosa and increased intestinal permeability. ${ }^{28}$

A high-fat diet dramatically increased intestinal permeability via a mechanism associated with reduced expression of epithelial tight junction proteins, including zonulin and occludin..$^{29}$ Cani et al demonstrated that prebiotic-treated mice exhibited lower plasma LPS and cytokines and decreased hepatic expression of inflammatory and oxidative stress markers. ${ }^{30}$ This decreased inflammatory tone was associated with lower intestinal permeability and improved tight junction integrity compared to controls. In these experiments, prebiotic supplementation increased endogenous intestinotrophic proglucagon-derived peptide (GLP-2) production, whereas the GLP-2 antagonist abolished most of the prebiotic effects.

GLP-2 plays a significant role in the adaptive regulation of bowel mass, mucosal integrity, stimulation of enterocyte proliferation and prevention of apoptosis. ${ }^{31}$ GLP-2's growth-promoting and cytoprotective properties in the gastrointestinal tract have aroused interest in its use as a therapeutic agent for the treatment of GI diseases involving malabsorption, inflammation and/or mucosal damage. It promotes expansion of the GI mucosal surface area, stimulates the uptake of luminal nutrients including carbohydrates and amino acids, enhances mucosal hexose transport, enhances the expression of genes encoding nutrient transporters and increases multiple enzymes involved in digestion along the GI tract. Interestingly, pharmacological GLP-2 treatment decreased gut permeability, systemic and hepatic inflammatory phenotype 
associated with obesity to a similar extent as that observed following prebiotic-induced changes in gut microbiota. ${ }^{30}$ In summary, a selective gut microbiota change can increase endogenous GLP-2 production and consequently improves gut barrier functions via a GLP-2-dependent mechanism, contributing to the improvement of gut barrier functions during obesity and diabetes. These data suggest that gut bacteria are involved in intestinal permeability control and in the occurrence of metabolic endotoxemia.

The endocannabinoid system

Several studies have suggested a close relationship between LPS, metabolic endotoxemia and the endocannabinoid (eCB) system.

Obesity and its metabolic complications are associated with macrophage infiltration, which is responsible for almost all adipose tissue TNF-alpha and IL-6 expression involved in inflammatory pathways. ${ }^{32}$ The activation of macrophages is dependent on LPS/ CD14. ${ }^{19}$ On the other hand, LPS regulates the synthesis of eCB in macrophages and obesity is associated with increased eCB plasma levels. ${ }^{33,34}$ The endocannabinoid system is composed of endogenous bioactive lipids that act through cannabinoid receptor 1 (CB1) and $2 .{ }^{35}$ Cannabinoid receptor 1 (CB1) blockage in obese mice improved gut barrier function by lowering alterations of tight junctions proteins (zonulin and occludin) and decreased plasma LPS levels. ${ }^{36}$ Cannabinoid agonist administration significantly increased LPS and endotoxemia through changes in permeability. ${ }^{36,37}$ Consequently, gut microbiota may have a critical function in the regulation of gut permeability, contributing to endotoxemia, through the endocannabinoid system and LPS regulatory loop.

The ECB/LPS system also appears to have an important role in adipose tissue plasticity. Adipocyte differentiation and lipogenesis is reduced in the presence of physiological levels of LPS, whereas eCB activation increased adipogenesis in lean mice under physiological conditions ${ }^{36}$ Surprisingly, specific modulation of gut microbiota and CB1 blockage increased adipocyte differentiation and lipogenesis. ${ }^{36}$ One possible hypothesis involves the fact that $\mathrm{CB} 1$ blockage reduces gut permeability, thus reducing LPS levels under pathophysiological conditions such as obesity, which counteracts their inhibitory effects on adipocyte differentiation and lipogenesis that might paradoxically increase. These data show that the LPS/eCB system regulatory loops contribute to deregulation of adipogenesis, thereby perpetuating the disequilibrium and leading to a vicious cycle in obesity.

Intestinal alkaline phosphatase

Intestinal alkaline phosphatase (IAP) has a pivotal role in intestinal homeostasis. IAP is known as a regulator of lipid absorption across the apical membrane of enterocytes. ${ }^{38}$ It also controls bacterial endotoxin-induced inflammation by dephosphoriylation, detoxifying intestinal LPS, thus acting as a host defense factor against LPS. ${ }^{39}$ IAP expression is not only modulated by dietary components, including fat, but also controlled by gut microbiota. ${ }^{40,41}$

Consumption of a high-fat diet in conjunction with an obese phenotype was associated with changes in the gut microbiota, a decrease in IFA, an increase in LPS and ileal inflammation. ${ }^{40}$ Ghoshal et al demonstrated that enterocytes internalize LPS from the apical surface and transport LPS to the Golgi complex. This complex also contains chylomicrons, the lipoproteins responsible for transport of dietary long-chain fat through blood and mesenteric lymph. It was observed that chylomicrons promote intestinal LPS absorption. ${ }^{42}$ Thus, excess chylomicron formation during high-fat feeding facilitates endotoxin translocation via a reduction in IAP activity, inducing intestinal inflammation that is present in obesity and insulin resistant states. ${ }^{43}$

\section{The 'storage' hypothesis}

Energy metabolism can be profoundly regulated by host gut microbiota, that is, microbiota modulates energy balance. Adult germ-free conventionalization with a normal microbiota collected from the cecum of conventionally raised mice produced a $60 \%$ increase in body fat and insulin resistance, despite reduced chow consumption. ${ }^{44}$ In this line, microbiota transplantation from the cecum of an obese donor $(\mathrm{ob} / \mathrm{ob})$ to adult germ-free mice resulted in a greater relative abundance of Firmicutes and a significant increase in body fat over two weeks, compared with mice colonized with a lean microbiota, despite no differences in food intake. ${ }^{9}$ As is well known, energy balance results from an equilibrium between energy 
intake and energy expenditure. The above experiments gave rise to the hypothesis that obesity-associated gut microbiome has an increased capacity for energy harvest from the diet, the so called 'storage effect' hypothesis. This hypothesis is based on the following probabilities: microbial fermentation of dietary polysaccharides that cannot be digested by the host, intestinal absorption of monosaccharides and lipid metabolism regulation by microbiota.

\section{Fermentation of dietary polysaccharides}

Gut microbiota fermentation degrades non-digestible polysaccharides into short-chain fatty acids (SCFA), including acetate, propionate and butyrate, and other subproducts in the cecum and colon. ${ }^{45} \mathrm{Gut}$ microbiota is enriched by many enzymes involved in processing these otherwise indigestible dietary polysaccharides for the metabolism of, among others, starch/sucrose, galactose and butanoate. ${ }^{9}$ As predicted from these metagenomic analyses, the ob/ob cecum had a higher concentration of the major fermentation end-products - butyrate and acetate. These findings are consistent with the fact that Firmicutes are butyrate producers. ${ }^{46,47}$ SCFAs are ligands for the G-protein coupled receptors 41 and 43 (GPR41 and GPR43). ${ }^{48}$ In concordance with previously published results, Samuel et al have shown that GPR41 knockout mice, colonized with a specific fermentative microbial community, were resistant to fat mass gain..$^{49}$ In addition, GPR43 knockout mice fed a high-fat diet presented significantly lower body fat mass and higher body lean mass compared to their littermate controls. These phenotype changes were accompanied by improved glucose control and lower HOMA índex. ${ }^{50}$ These data emphasize the potential role of GPRs and SCFA in fat mass development and regulation of glucose metabolism.

\section{Intestinal absorption of monosaccharides}

Conventionalization of germ-free mice results in a two-fold increase of the capillary density in the villus epithelium of the small intestine. ${ }^{51}$ Thus, gut microbiota shape the intestinal villus microvasculature, which increases the intestine's absorptive capacity. ${ }^{51}$ Experimental data corroborate these observations, demonstrating that gut microbiota stimulates monosaccharides absorption from the gut lumen. ${ }^{44}$
Furthermore, ob/ob mice also have less energy in their feces relative to their lean littermates. ${ }^{9}$

\section{Lipid metabolism regulation}

There is strong evidence that gut microbiota is a regulator of fat storage. A 14-d conventionalization of GF mice resulted in a 2.3 -fold increase in liver triglyceride content and was accompanied by statistically significant increase in liver acetyl-CoA carboxylase and fatty acid synthase, two key enzymes in the de novo fatty acid biosynthetic pathway, known targets of sterol response element binding protein 1 (SREBP-1) and carbohydrate response element binding protein (ChREBP) ${ }^{44}$ Moreover, it was concluded that increased capacity of storage triglycerides in adipocytes was mediated through the suppression of intestinal expression of a circulating lipoprotein lipase inhibitor. Lipoprotein lipase is a key regulator of fatty acid release from triglyceride-rich lipoproteins; in turn, adipocyte lipoprotein lipase activity is associated with an increase in fatty acids uptake and adipocyte triglyceride accumulation. FIAF (fasting-induced adipose factor), also named angiopoietin-like protein 4 , is an inhibitor of lipoprotein lipase activity, suppressed in conventionalized germ-free mice, which explains the increased triglycerides storage in adipocytes in these mice. It therefore seems that microbiota coordinates increased hepatic lipogenesis, promoting storage of calories from the diet as fat, through suppression of FIAF, and resulting in increased lipoprotein lipase activity in adipocytes.

Furthermore, gut microbiota seems to be an active player in fatty acid oxidation. Besides the observation that germ-free knockout mice lacking FIAF were not protected against obesity, Backed et al demonstrated that germ-free mice were protected against obesity, despite consuming a western, high-fat, sugar-rich diet. $^{52}$ This lean phenotype was related to increase skeletal muscle and liver levels of phosphorylated AMP-activated protein kinase and its downstream targets - acetylCoA carboxylase, carnitine-palmitoyltransferase - that are involved in fatty acid oxidation.

\section{Gut microbiota modulation}

Understanding the metabolic impact of the complex interaction between gut microbiota and the host has driven interest in manipulating microbiota in order 
to develop new therapeutic targets for the metabolic diseases, obesity and T2D

\section{Gut microbiota transplantation}

As has been described, gut microbiota profoundly affects metabolic homeostasis. Microbiota transplantation from mice fed a diet that induced obesity to lean germ-free mice promoted greater fat increase than transplants from lean donors. ${ }^{53}$ The main principle of fecal transplant is therefore the possibility of this procedure replacing pathogenic microbes by beneficial communities, thus restoring the gut microbiota balance and enabling the cure of the disease. Although gut microbiota transplantation is a controversial issue, the metabolic effects of transplanting intestinal microbiota from lean donors to male recipients with metabolic syndrome were recently assessed. ${ }^{54}$ Individuals were randomly assigned to receive intestinal infusions of allogenic or autologous microbiota. Six weeks after, subjects that received infusions of microbiota from lean donors had an insulin sensitivity increase, along with increase of butyrate-producing intestinal microbiota.

\section{Bariatric surgery}

Bariatric surgery, and specifically Roux-en-Y gastric bypass (RYGB), has become a promising treatment which, besides promoting weight loss, can also improve or resolve T2D in severely obese patients. ${ }^{55,56}$ Bariatric surgery seems, furthermore, an effective model to study the gut microbiota profile and its association with beneficial metabolic/inflammatory effects underlying the procedure, including loss of weight and resolution of T2D.

Zhang et al analyzed gut microbiota of 9 individuals, 3 in each of the categories of normal weight, obese and after gastric bypass surgery. ${ }^{57}$ It was found that Firmicutes were dominant in normal-weight and obese individuals, but significantly decreased in those post bypass. After 3 months (M3) of RYGB, the Bacteroides/Prevotella group had increased in previously obese individuals. Escherichia coli species had also increased at M3 and were inversely correlated with fat mass and leptin levels independently of changes in food intake. ${ }^{11}$ Interestingly, gut microbiota richness was also improved after RYGB and 202 white adipose tissue (WAT) genes changed following RYGB. ${ }^{58}$ Furthermore, variations of gut microbiota were associated with changes in WAT gene expression. To test the hypothesis that some of the effects of RYGB were caused by changes of the host-microbiota relationship, Liou et al used a mouse model of RYGB. Transferal of the gut microbiota from RYGB mice to non-operated ones resulted in weight loss and decreased fat mass, this corroborating the present hypotheses concerning the impact of gut microbiota modifications after RYGB in weight loss. ${ }^{59}$

Although the available studies seem to bear out the relationship between gut microbiota modulation and underlying metabolic improvement after bariatric surgery, it is still necessary to confirm these observations in larger cohorts. It is also of great importance to understand whether microbiota changes are the cause or the consequence of weight loss and glycemic control after bariatric surgery.

\section{Prebiotics}

The concept of prebiotics was first introduced in 1995 by Gibson and Roberfroid. ${ }^{60}$ A prebiotic is a selectively fermented ingredient that results in specific changes in the composition and/or activity of the gastrointestinal microbiota, thus conferring benefit(s) upon host health, this especially applying to Bifidobacterium and Lactobacillus. ${ }^{61}$ Gut microbiota enables the fermentation of these non-digestible compounds so as to produce short-chain fatty acids. ${ }^{62}$

High-fat feeding changes gut microbiota towards a reduced level of Bifidobacterium and increases metabolic endotoxemia, thus resulting in body weight gain and diabetes. ${ }^{19}$ Accordingly, feeding mice with a prebiotic, oligofructose, restored the levels of Bifidobacteria and normalized endotoxemia. Importantly, Bifidobacterium species were negatively correlated with endotoxemia. ${ }^{20}$ In humans, oligofructose supplementation also promoted weight loss and improved glucose regulation in overweight adults. ${ }^{63}$ Furthermore, it increased satiety and reduced hunger. ${ }^{64}$ Thus, the mechanisms linking prebiotic-induced changes in gut microbiota and their beneficial metabolic effects seem to go beyond the reduction of metabolic endotoxemia.

Oligofructose supplementation decreased food intake, fat mass development and hepatic steatosis in normal and in obese rats; furthermore, it exerts / exerted an antidiabetic effect in streptozotocin-treated rats and high-fat-fed mice. The beneficial effects of 
this prebiotic were associated with an increase in the number of L cells and of glucagon-like peptide-1 (GLP-1). Moreover, it was also found that oligofructose was able to modulate PYY and ghrelin, which could be involved in the control of food intake. ${ }^{65}$ The relationship between the beneficial metabolic effects of prebiotics and GLP-1 were also supported by other studies. ${ }^{66,67}$ Endogenous intestinotrophic proglucagon-derived peptide (GLP-2) has also demonstrated its clinical relevance in association with the use of prebiotics. Cani et al have shown that prebiotic-fed mice had lower levels of several plasma cytokines, such as TNF $\alpha$, IL1b, IL1 $\alpha$, IL6, INF $\gamma$, known to promote tight junction disruption and a significant decrease of the chemokines MCP-1 and MIP-1 $1^{\text {a }} .{ }^{68,69}$ The decrease in these inflammatory markers was associated with improved tight junction integrity and lower intestinal permeability, which in turn resulted in lower LPS levels. In addition, GLP-2 production was increased, while the GLP-2 antagonist abolished the beneficial effects of prebiotics. ${ }^{68}$ These data raised the hypothesis that a selective gut microbiota increases endogenous GLP-2 and consequently improves gut barrier integrity by a GLP-2-dependent mechanism, contributing to the improvement of the gut barrier in obesity and diabetes.

In humans, oligofructose supplementation also promoted beneficial metabolic effects, including weight loss and improved glucose regulation in overweight adults, these being associated with suppressed ghrelin and enhanced PYY. ${ }^{70}$ Recently, T2D patients were supplemented with transglucosidase, resulting in a decrease in plasma glucose levels and in an increase in Bacteroidetes-to-Firmicutes ratio. ${ }^{71}$ However, there are conflicting data regarding prebiotic effects in humans. ${ }^{72,73}$

It now appears that other constituents, besides the non-digestible carbohydrates of prebiotics, influence gut microbiota modulation, as shown by Isken et al. Indeed, the solubility of dietary fibres (ratio of soluble vs insoluble), when added to a high-fat western-style diet, differently affected body weight and insulin sensitivity in obesity-prone mice. ${ }^{74}$

Regarding the presented data, nutritional approaches with prebiotics thus appear to be an important target to consider in the management of obesity and T2D.
However, it is essential to investigate which components may affect gut microbiota modulation and its impact on metabolic modulation.

\section{Probiotics}

Probiotics are food supplements that contain living bacteria, in particular those of the genera Lactobacillus and Bifidobacterium which act through diverse mechanisms to confer a health benefit to the host. ${ }^{75}$ Probiotics appear to benefit T2D and obesity. However, a better insight into the bacterial strains and host metabolism is necessary for their efficient use. Rodent models have provided robust evidence for identifying human gut commensals that could be developed as probiotic therapies. Several studies have addressed the metabolic effects of Bifidobacterium supplementation. For instance, it was demonstrated that feeding Bifidobacterium improved several aspects of the metabolic syndrome reducing endotoxin concentration and intestinal inflammation. ${ }^{76,77}$ However, these effects seem to be strain-dependent, which requires a detailed understanding of each strain and the way it affects fat and glucose metabolism. ${ }^{78}$ As concerns Lactobacillus supplementation, although there is experimental data showing its effects in reduction of body weight, ${ }^{79,80}$ some studies do not report these differences. Recently, Akkermansia muciniphila was shown to be decreased in obese and T2D mice. ${ }^{81}$ This study found that $A$. muciniphila treatment reversed high-fat diet-induced metabolic disorders, including fat-mass gain, metabolic endotoxemia, adipose tissue inflammation and insulin resistance. Moreover, A. muciniphila administration increased the intestinal levels of endocannabinoids, which, as has been previously described, control inflammation and the gut barrier. ${ }^{81}$ In humans there are increasingly well-controlled studies that seek to demonstrate the suggested potential benefits of probiotics in weight and diabetes management. ${ }^{82-85}$

\section{Antibiotics}

Antibiotics are agents that inhibit the growth of microorganisms. Administration of antimicrobial agents is another viable option for modulation of the gut microbiota profile. Regarding the fact that gut microbiota has been linked to obesity and T2D pathogenesis, Membrez et al studied the effects of norfloxacin and ampicillin in ob/ob mice. ${ }^{86}$ After 
a 2-week intervention, a significant improvement in fasting glycemia and oral glucose tolerance was observed. Concomitant reduction of plasma LPS and increase of adiponectin further supported the antidiabetic effects of the antibiotic treatment. ${ }^{86}$ In high-fat fed mice, the modulation of gut microbiota by antibiotics administration resulted in improved glucose tolerance by reducing LPS levels and inflammatory status. In addition, it also increased the circulating levels of acetate, which activates AMPK and reduces macrophage infiltration. ${ }^{87}$ Moreover, vancomycintreated high-fat fed mice gained less weight after the treatment and had lower fasting blood glucose and plasma TNF $\alpha$ compared to the obese controls. ${ }^{88}$ Importantly, in a NOD mouse model for diabetes treated with vancomycin, although the cumulative incidence of diabetes was lower, one single species, Akkermansia muciniphila, became dominant. ${ }^{89}$

In humans, however, clinical studies are mainly limited to the characterization of the composition of gut microbiota after antibiotics administration..$^{90-92}$ However, there are some data that link antibiotic exposure to obesity risk. ${ }^{93-95}$ Thus, while in mice models the available data suggest that gut microbiota is a realistic target, in humans it remains uncertain whether antibiotics use might be associated with metabolic improvement.

\section{CONCLUSION}

Gut microbiota provides a broad field of increasing importance for the comprehension of obesity and T2D. As has been shown, this comprehension may make possible the development of new specific targets for the improvement, or even cure, of these prevalent metabolic diseases.

\section{DISCLOSURE}

There is no conflict of interest that would undermine the impartiality of this scientific work. There is no potential conflict of interest by any author of manuscript.

\section{REFERENCES}

1. WHO | Obesity and overweight. [cited 2014 Feb 8]; Available from: http:/www.who.int/mediacentre/fact- sheets/fs311/en/

2. WHO | Diabetes. [cited 2014 Feb 8]; Available from: http://www.who.int/mediacentre/factsheets/fs312/en/

3. Cani PD, Delzenne NM, 2009 The role of the gut microbiota in energy metabolism and metabolic disease. Curr Pharm Des 15: 1546-1558.

4. Qin J, Li R, Raes J, et al, 2010 A human gut microbial gene catalogue established by metagenomic sequencing. Nature 464: 59-65.

5. Prakash S, Rodes L, Coussa-Charley M, Tomaro-Duchesneau C, 2011 Gut microbiota: next frontier in understanding human health and development of biotherapeutics. Biologics 5: 71-86.

6. Bäckhed F, Ley RE, Sonnenburg JL, Peterson DA, Gordon JI, 2005 Host-bacterial mutualism in the human intestine. Science 307: 1915-1920.

7. Prakash S, Rodes L, Coussa-Charley M, TomaroDuchesneau C, 2011 Gut microbiota: next frontier in understanding human health and development of biotherapeutics. Biologics 5: 71-86.

8. Ley RE, Bäckhed F, Turnbaugh P, Lozupone CA, Knight RD, Gordon JI, 2005 Obesity alters gut microbial ecology. Proc Natl Acad Sci U S A 102: 11070-11075.

9. Turnbaugh PJ, Ley RE, Mahowald MA, Magrini V, Mardis ER, Gordon JI, 2006 An obesity-associated gut microbiome with increased capacity for energy harvest. Nature 444: 1027-1031.

10. Ley RE, Turnbaugh PJ, Klein S, Gordon JI, 2006 Microbial ecology: human gut microbes associated with obesity. Nature 444: 1022-1023.

11. Furet J, Kong L, Tap J, et al, 2010 Differential Adaptation of Human Gut Microbiota to bariatric surgery-induced weight loss: links with metabolic and low-grade inflammation markers. Diabetes 59: 3049-3057.

12. Kong LC, Wuillemin P-H, Bastard J-P, et al, 2013 Insulin resistance and inflammation predict kinetic body weight changes in response to dietary weight loss and maintenance in overweight and obese subjects by using a Bayesian network approach. Am J Clin Nutr 98: 1385-1394.

13. Le Chatelier E, Nielsen T, Qin J, et al, 2013 Richness of human gut microbiome correlates with metabolic markers. Nature 500: 541-546.

14. Geurts L, Lazarevic V, Derrien M, et al, 2011 Altered gut microbiota and endocannabinoid system tone in obese and diabetic leptin-resistant mice: impact on apelin regulation in adipose tissue. Front Microbiol 2: 1-17.

15. Larsen N, Vogensen FK, van den Berg FWJ, et al, 2010 Gut microbiota in human adults with type 2 diabetes differs from non-diabetic adults. PLoS One 5: e9085.

16. Qin J, Li Y, Cai Z, et al, 2012 A metagenome-wide association study of gut microbiota in type 2 diabetes. Nature 490: 55-60.

17. Karlsson FH, Tremaroli V, Nookaew I, et al, 2013 Gut metagenome in European women with normal, impaired 
and diabetic glucose control. Nature 498: 99-103.

18. Amar J, Serino M, Lange C, et al, 2011 Involvement of tissue bacteria in the onset of diabetes in humans: evidence for a concept. Diabetologia 54: 3055-3061.

19. Cani PD, Amar J, Iglesias MA, et al, 2014 Metabolic endotoxemia initiates obesity and insulin resistance. Diabetes 56: 1761-1772.

20. Cani PD, Neyrinck AM, Fava F, et al, 2007 Selective increases of bifidobacteria in gut microflora improve high-fat-diet-induced diabetes in mice through a mechanism associated with endotoxaemia. Diabetologia 50: 2374-2383.

21. Laugerette F, Vors C, Géloën A, et al, 2011 Emulsified lipids increase endotoxemia: possible role in early postprandial low-grade inflammation. J Nutr Biochem 22: 53-59.

22. Poggi M, Bastelica D, Gual P, et al, 2007 C3H/HeJ mice carrying a toll-like receptor 4 mutation are protected against the development of insulin resistance in white adipose tissue in response to a high-fat diet. Diabetologia 50: 1267-1276.

23. Fasano A, 2011 Zonulin and its regulation of intestinal barrier function: the biological door to inflammation, autoimmunity, and cancer. Physiol Rev 91: 151-175.

24. Sonier B, Patrick C, Ajjikuttira P, Scott FW, 2009 Intestinal immune regulation as a potential diet-modifiable feature of gut inflammation and autoimmunity. Int Rev Immunol 28: 414-445.

25. Groschwitz KR, Hogan SP, 2009 Intestinal barrier function: molecular regulation and disease pathogenesis. $\mathrm{J}$ Allergy Clin Immunol 124: 3-22.

26. Lamprecht M, Bogner S, Schippinger G, et al, 2012 Probiotic supplementation affects markers of intestinal barrier, oxidation, and inflammation in trained men; a randomized, double-blinded, placebo-controlled trial. J Int Soc Sports Nutr 9: 45.

27. Xiao S, Fei N, Pang X, et al, 2014 A gut microbiotatargeted dietary intervention for amelioration of chronic inflammation underlying metabolic syndrome. FEMS Microbiol Ecol 87: 357-367.

28. Klaus DA, Motal MC, Burger-Klepp U, et al, 2013 Increased plasma zonulin in patients with sepsis. Biochem medica 23: 107-111.

29. Cani PD, Bibiloni R, Knauf C, et al, 2008 Changes in gut microbiota control metabolic endotoxemia-induced inflammation in high-fat diet-induced obesity and diabetes in mice. Diabetes 57: 1470-1481.

30. Cani PD, Possemiers S, Van De Wiele T, et al, 2009 Changes in gut microbiota control inflammation in obese mice through a mechanism involving GLP-2- driven improvement of gut permeability. Gut 58: 1091-1103.

31. Estall JL, Drucker DJ, 2006 Glucagon-like Peptide-2. Annu Rev Nutr 26: 391-411.

32. Weisberg SP, McCann D, Desai M, Rosenbaum M, Leibel RL, Ferrante AW, 2003 Obesity is associated with macrophage accumulation in adipose tissue. $\mathrm{J}$
Clin Invest 112: 1796-1808.

33. Blüher M, Engeli S, Klöting N, et al, 2006 Dysregulation of the peripheral and adipose tissue endocannabinoid system in human abdominal obesity. Diabetes 55: 3053-3060.

34. Côté M, Matias I, Lemieux I, et al, 2007 Circulating endocannabinoid levels, abdominal adiposity and related cardiometabolic risk factors in obese men. Int J Obes 31: 692-699.

35. Silvestri C, Di Marzo V, 2013 The endocannabinoid system in energy homeostasis and the etiopathology of metabolic disorders. Cell Metab 17: 475-490.

36. Muccioli GG, Naslain D, Bäckhed F, et al, 2010 The endocannabinoid system links gut microbiota to adipogenesis. Mol Syst Biol 6: 392.

37. Alhamoruni A, Wright KL, Larvin M, O'Sullivan SE, 2012 Cannabinoids mediate opposing effects on inflammation-induced intestinal permeability. Br J Pharmacol 165: 2598-2610.

38. Lallès J-P, 2010 Intestinal alkaline phosphatase: multiple biological roles in maintenance of intestinal homeostasis and modulation by diet. Nutr Rev 68: 323-332.

39. Koyama I, Matsunaga T, Harada T, Hokari S, Komoda T, 2002 Alkaline phosphatases reduce toxicity of lipopolysaccharides in vivo and in vitro through dephosphorylation. Clin Biochem 35: 455-461.

40. De La Serre CB, Ellis CL, Lee J, Hartman AL, Rutledge JC, Raybould HE, 2010 Propensity to high-fat dietinduced obesity in rats is associated with changes in the gut microbiota and gut inflammation. Am J Physiol 299: G440-448.

41. Bates JM, Akerlund J, Mittge E, Guillemin K, 2004 Intestinal alkaline phosphatase detoxifies lipopolysaccharide and prevents inflammation in zebrafish in response to the gut microbiota. Cell Host Microbe 2: 371-382.

42. Ghoshal S, Witta J, Zhong J, de Villiers W, Eckhardt E, 2009 Chylomicrons promote intestinal absorption of lipopolysaccharides. J Lipid Res 50: 90-97.

43. Ding S, Chi MM, Scull BP, et al, 2010 High-fat diet: bacteria interactions promote intestinal inflammation which precedes and correlates with obesity and insulin resistance in mouse. PLoS One 5: e12191.

44. Ding H, Wang T, Hooper L V, et al, 2004 The gut microbiota as an environmental factor that regulates fat storage. Proc Natl Acad Sci U S A 101: 15718-15712.

45. Louis P, Scott KP, Duncan SH, Flint HJ, 2007 Understanding the effects of diet on bacterial metabolism in the large intestine. J Appl Microbiol 102:1197-1208.

46. Louis P, Flint HJ, 2009 Diversity, metabolism and microbial ecology of butyrate-producing bacteria from the human large intestine. FEMS Microbiol Lett 294: $1-8$.

47. Barcenilla A, Pryde SE, Martin JC, et al, 2000 Phylogenetic relationships of butyrate-producing bacteria from the human gut. Appl Environ Microbiol 66: 1654-1661. 
48. Le Poul E, Loison C, Struyf S, et al, 2003 Functional characterization of human receptors for short chain fatty acids and their role in polymorphonuclear cell activation. J Biol Chem 278: 25481-25489.

49. Samuel BS, Shaito A, Motoike T, et al, 2008 Effects of the gut microbiota on host adiposity are modulated by the short-chain fatty-acid binding $G$ protein-coupled receptor, Gpr41. Proc Natl Acad Sci U S A 105: 16767-16772.

50. Bjursell M, Admyre T, Göransson M, et al, 2011 Improved glucose control and reduced body fat mass in free fatty acid receptor 2-deficient mice fed a high-fat diet. Am J Physiol Endocrinol Metab 300: E211-220.

51. Stappenbeck TS, Hooper LV, Gordon JI, 2002 Developmental regulation of intestinal angiogenesis by indigenous microbes via Paneth cells. Proc Natl Acad Sci U S A 99: 15451-15455.

52. Bäckhed F, Manchester JK, Semenkovich CF, Gordon JI, 2007 Mechanisms underlying the resistance to dietinduced obesity in germ-free mice. Proc Natl Acad Sci U S A 104: 979-984.

53. Turnbaugh PJ, Bäckhed F, Fulton L, Gordon JI, 2008 Diet-Induced Obesity Is Linked to Marked but Reversible Alterations in the Mouse Distal Gut Microbiome. Cell Host Microbe 3: 213-223.

54. Vrieze A, Van Nood E, Holleman F, et al, 2012 Transfer of intestinal microbiota from lean donors increases insulin sensitivity in individuals with metabolic syndrome. Gastroenterology 143: 913-916.

55. Mingrone G, Panunzi S, De Gaetano A, et al, 2012 Bariatric surgery versus conventional medical therapy for type 2 diabetes. N Engl J Med 366: 1577-1585.

56. Schauer BR, Kashyap SR, Wolski k, et al, 2012 Bariatric Surgery versus Intensive Medical Therapy in Obese Patients with Diabetes. N Engl J Med 366: 1567-1576.

57. Zhang H, DiBaise JK, Zuccolo A, et al, 2009 Human gut microbiota in obesity and after gastric bypass. Proc Natl Acad Sci U S A 106: 2365-2370.

58. Kong L-C, Tap J, Aron-Wisnewsky J, et al, 2013 Gut microbiota after gastric bypass in human obesity: increased richness and associations of bacterial genera with adipose tissue genes. Am J Clin Nutr 98: 16-24.

59. Liou AP, Paziuk M, Luevano J-M, Machineni S, Turnbaugh PJ, Kaplan LM, 2013 Conserved shifts in the gut microbiota due to gastric bypass reduce host weight and adiposity. Sci Transl Med 5: 178ra41.

60. Gibson GR, Roberfroid MB, 1995 Dietary modulation of the human colonic microbiota: introducing the concept of prebiotics. J Nutr 125: 1401-1412.

61. Roberfroid M, Gibson GR, Hoyles L, et al, 2010 Prebiotic effects: metabolic and health benefits. Br J Nutr 104: Suppl 2: 1-63.

62. Soldavini J, Kaunitz JD, 2013 Pathobiology and potential therapeutic value of intestinal short-chain fatty acids in gut inflammation and obesity. Dig Dis Sci 58: 2756-2766.
63. Parnell JA, Reimer RA, 2009 Weight loss during oligofructose supplementation is associated with decreased ghrelin and increased peptide YY in overweight and obese adults. Am J Clin Nutr 89: 1751-1759.

64. Cani PD, Joly E, Horsmans Y, Delzenne NM, 2006 Oligofructose promotes satiety in healthy human: a pilot study. Eur J Clin Nutr 60: 567-572.

65. Delzenne NM, Cani PD, Neyrinck AM, 2007 Modulation of glucagon-like peptide 1 and energy metabolism by inulin and oligofructose: Experimental Data. J Nutr 137: Suppl 11: 2547-2551.

66. Cani PD, 2006 Improvement of glucose tolerance and hepatic insulin sensitivity by oligofructose requires a functional glucagon-like peptide 1 receptor. Diabetes 55: $1484-1490$.

67. Cani PD, Dewever C, Delzenne NM, 2004 Inulin-type fructans modulate gastrointestinal peptides involved in appetite regulation (glucagon-like peptide-1 and ghrelin) in rats. Br J Nutr 92: 521-526.

68. Cani PD, Possemiers S, Van de Wiele T, et al, 2009 Changes in gut microbiota control inflammation in obese mice through a mechanism involving GLP-2-driven improvement of gut permeability. Gut 58: 1091-1103.

69. Capaldo CT, Nusrat A, 2009 Cytokine regulation of tight junctions. Biochim Biophys Acta 1788: 864-871.

70. Parnell JA, Reimer RA, 2009 Weight loss during oligofructose supplementation is associated with decreased ghrelin and increased peptide YY in overweight and obese adults. Am J Clin Nutr 89: 1751-1759.

71. Sasaki M, Ogasawara N, Funaki Y, et al, 2013 Transglucosidase improves the gut microbiota profile of type 2 diabetes mellitus patients: a randomized double-blind, placebo-controlled study. BMC Gastroenterol 13: 81.

72. Weickert MO, Arafat AM, Blaut M, et al, 2011 Changes in dominant groups of the gut microbiota do not explain cereal-fiber induced improvement of whole-body insulin sensitivity. Nutr Metab 8: 90.

73. Lappi J, Salojärvi J, Kolehmainen M, et al, 2013 Intake of whole-grain and fiber-rich rye bread versus refined wheat bread does not differentiate intestinal microbiota composition in Finnish adults with metabolic syndrome. J Nutr 143: 648-655.

74. Isken F, Klaus S, Osterhoff M, Pfeiffer AFH, Weickert MO, 2010 Effects of long-term soluble vs. insoluble dietary fiber intake on high-fat diet-induced obesity in C57BL/6J mice. J Nutr Biochem 21: 278-284.

75. Johnson BR, Klaenhammer TR, 2014 Impact of genomics on the field of probiotic research: historical perspectives to modern paradigms. Antonie Van Leeuwenhoek 106: 141-156.

76. Chen J, Wang R, Li X-F, Wang R-L, 2012 Bifidobacterium adolescentis supplementation ameliorates visceral fat accumulation and insulin sensitivity in an experimental model of the metabolic syndrome. Br J Nutr 107: 1429-1434.

77. Chen JJ, Wang R, Li X, Wang R, 2011 Bifidobacte- 
rium longum supplementation improved high-fat-fedinduced metabolic syndrome and promoted intestinal Reg I gene expression. Exp Biol Med (Maywood) 236: 823-831.

78. Yin Y-N, Yu Q-F, Fu N, Liu X-W, Lu F-G, 2010 Effects of four Bifidobacteria on obesity in high-fat diet induced rats. World J Gastroenterol 16: 3394-3401.

79. Miyoshi M, Ogawa A, Higurashi S, Kadooka Y, 2014 Anti-obesity effect of Lactobacillus gasseri SBT2055 accompanied by inhibition of pro-inflammatory gene expression in the visceral adipose tissue in diet-induced obese mice. Eur J Nutr 53: 599-606.

80. Park D-Y, Ahn Y-T, Park S-H, et al, 2013 Supplementation of Lactobacillus curvatus HY7601 and Lactobacillus plantarum KY1032 in diet-induced obese mice is associated with gut microbial changes and reduction in obesity. PLoS One 8: e59470.

81. Everard A, Belzer C, Geurts L, et al, 2013 Cross-talk between Akkermansia muciniphila and intestinal epithelium controls diet-induced obesity. Proc Natl Acad Sci 110: 9066-9071.

82. Asemi Z, Zare Z, Shakeri H, Sabihi S-S, Esmaillzadeh A, 2013 Effect of multispecies probiotic supplements on metabolic profiles, hs-CRP, and oxidative stress in patients with type 2 diabetes. Ann Nutr Metab 63: 1-9.

83. Ogawa A, Kadooka Y, Kato K, Shirouchi B, Sato M, 2014 Lactobacillus gasseri SBT2055 reduces postprandial and fasting serum non-esterified fatty acid levels in Japanese hypertriacylglycerolemic subjects. Lipids Health Dis 13: 36.

84. Kadooka Y, Sato M, Ogawa A, et al, 2013 Effect of Lactobacillus gasseri SBT2055 in fermented milk on abdominal adiposity in adults in a randomised controlled trial. Br J Nutr 110: 1696-1703.

85. Arora T, Anastasovska J, Gibson G, et al, 2012 Effect of Lactobacillus acidophilus NCDC 13 supplementation on the progression of obesity in diet-induced obese mice. Br J Nutr 108: 1382-1389.

86. Membrez M, Blancher F, Jaquet M, et al, 2008 Gut microbiota modulation with norfloxacin and ampicillin enhances glucose tolerance in mice. FASEB J 22: 2416-2426.

87. Carvalho BM, Guadagnini D, Tsukumo DML, et al, 2012 Modulation of gut microbiota by antibiotics improves insulin signalling in high-fat fed mice. Diabetologia 55: 2823-2834.

88. Murphy EF, Cotter PD, Hogan A, et al, 2013 Divergent metabolic outcomes arising from targeted manipulation of the gut microbiota in diet-induced obesity. Gut 62: 220-226.

89. Hansen CHF, Krych L, Nielsen DS, et al, 2012 Early life treatment with vancomycin propagates Akkermansia muciniphila and reduces diabetes incidence in the NOD mouse. Diabetologia 55: 2285-2294.

90. O’Sullivan O, Coakley M, Lakshminarayanan B, et al, 2013 Alterations in intestinal microbiota of elderly Irish subjects post-antibiotic therapy. J Antimicrob Chemother 68: 214-221.

91. Jernberg C, Löfmark S, Edlund C, Jansson JK, 2010 Long-term impacts of antibiotic exposure on the human intestinal microbiota. Microbiology 156: 3216-3223.

92. Jernberg C, Löfmark S, Edlund C, Jansson JK, 2007 Long-term ecological impacts of antibiotic administration on the human intestinal microbiota. ISME J 1: 56-66.

93. Thuny F, Richet H, Casalta J-P, Angelakis E, Habib G, Raoult D, 2010 Vancomycin treatment of infective endocarditis is linked with recently acquired obesity. PLoS One 5: e9074.

94. Ajslev TA, Andersen CS, Gamborg M, Sørensen TIA, Jess T, 2011 Childhood overweight after establishment of the gut microbiota: the role of delivery mode, prepregnancy weight and early administration of antibiotics. Int J Obes 35: 522-529.

95. Hernández E, Bargiela R, Diez MS, et al, 2013 Functional consequences of microbial shifts in the human gastrointestinal tract linked to antibiotic treatment and obesity. Gut Microbes 4: 306-315. 